\section{FRI0697 FRENCH NATIONWIDE SURVEY OF CHRONIC PAIN PERCEPTION IN 1739 PATIENTS WITH CHRONIC INFLAMMATORY RHEUMATISM}

A.-P. Trouvin ${ }^{1}$, C. Beller ${ }^{2}$, P. Preiss ${ }^{2}$, S. Perrot ${ }^{3} \cdot{ }^{1}$ Rheumatology, Hopitaux Universitaire Paris Centre - Cochin; ${ }^{2}$ Association AFPric; ${ }^{3}$ Pain Center, Hopitaux Universitaire Paris Centre - Cochin, Paris, France

Background: Pain is a major symptom in chronic inflammatory rheumatism. Pain intensity doesn't always correlate with disease activity and it can persist even when RA seems in clinical and biological remission. Little is known about the patients' perception of their treatment efficacy on pain, of pain influence in their life and their expectations for pain management. A French patients association (AFPric) conducted a national survey to explore patient's point of view on pain in their rheumatism.

Objectives: Describe in patients with chronic inflammatory rheumatism, their perception of treatment efficacy on pain, the impact of pain in their life and their expectations on pain care, in a nationwide survey.

Methods: A nationwide survey with a 20 -item questionnaire was conducted. The questions were developed by patients and rheumatologists in focus groups. Questionnaires were e-mailed to every member of the association (9065 members). Answers were collected until the 17th July 2016. Answers were anonymous.

Results: One thousand thirty nine patients (response rate 19.2\%) answered the questionnaire with 1510 women $(86.8 \%)$, mean age was 59 years [18-85 years]. For more than half $(58 \%)$ of the patients, their rheumatism had more than 10 years of evolution. Rheumatoid arthritis was the main rheumatism with 1377 patients $(87 \%)$. Among the 1194 patients $(76 \%)$ under conventional DMARDs $46.4 \%$ considered the cDMARDs efficacy on pain was between 70 and $100 \%$, on the other hand for $17.7 \%$ of the patients, cDMARDs efficacy on pain was less than $30 \%$. Among the 744 patients $(47.6 \%)$ receiving a biological DMARD, $66.2 \%$ considered bDMARDs efficacy on pain was between 70 and $100 \%$ and $10 \%$ considered it was $30 \%$ or less. Among the 658 patients $(42.3 \%)$ receiving oral corticosteroids, $56.6 \%$ considered corticosteroids' efficacy on pain between 70 and $100 \%$ and $12 \%$ considered it was less than $30 \%$. Patients were asked to rate the weight of pain among their symptoms, for $38 \%$ of the patients pain represents 70 to $100 \%$ of the symptoms of their rheumatism, for $31.7 \%$ it represents 40 to $60 \%$ and for $30.3 \% 30 \%$ or less. The mean weight is $51.55 \%$ Almost half of the patients $(46.8 \%)$ consider that their pain is underestimated by health professionals. For $37.2 \%$ of the patients, their current treatments are not appropriate for their current pain intensity. Finally $528(35.7 \%)$ patients are not satisfied with pain management offered by health professionals, $90 \%$ of them think that pain management is too standardized and $80 \%$ think that functional impact of chronic pain is not taken in consideration. Among the expectations, $95 \%$ of the patients wish a tailored pain management by their rheumatologist, $82.9 \%$ wish to participate to support groups with health professionals specialized in pain care.

Conclusions: This nationwide survey on pain among chronic inflammatory rheumatism patients shows that even in the biological DMARDs era, pain is the main concerning symptom for the patients. It is striking in this large cohort that almost half of the patients consider their pain insufficiently taken care of Acknowledgements: This study received an institutional grant from UCB Disclosure of Interest: None declared

DOI: 10.1136/annrheumdis-2017-eular.5781

\section{FRI0698 PREVOTELLA AND ALLOPREVOTELLA SPECIES CHARACTERIZE THE ORAL MICROBIOME OF EARLY RHEUMATOID ARTHRITIS}

B. Wolff ${ }^{1}$, S. Boutin ${ }^{2}$, H.-M. Lorenz ${ }^{3}$, H. Ueffing ${ }^{1}$, A. Dalpke ${ }^{2}$, D. Wolff ${ }^{1}$ ${ }^{1}$ Department of Conservative Dentistry, School of Dental Medicine, University Hospital Heidelberg; ${ }^{2}$ Dept. of Infectious Diseases-Medical Microbiology and Hygiene, University Hospital Heidelberg; ${ }^{3}$ Division of Rheumatology, Department of Medicine V, Ruprecht Karls University, Heidelberg, Germany

Background: We previously showed that in this early rheumatoid arthritis (ERA) cohort with a mean disease duration of $<6$ months, a clinically significant loss of clinical attachment loss as surrogate of alveolar bone loss is detectable compared with a matched healthy control cohort (1). Evidence is accumulating that distinct pathogens residing in reservoirs such as the oral cavity, the lung or the gut may play a role in driving the pathogenesis of RA (2-3).

Objectives: To characterize the oral microbiome associated with ERA.

Methods: 16S amplicon sequencing was used to analyze 88 samples of the supragingival and subgingival microbiome of 22 patients with ERA and 22 matched healthy controls. Oral and periodontal status, clinical activity of ERA and periodontitis, and socio-demographic parameters were used as explanatory variables in the next generation DNA sequencing analysis.

Results: Overall, a total of 4.702.161 16S RNA high-quality sequences were yielded. Using a distance-based similarity of $>97 \%$ for species-level operational taxonomic unit (OTU) assignment, a total of 1054 OTUs were identified (Fig 1). The oral microbiota was equally rich and diverse in ERA and control group. Subgingivally, Prevotella oris, Prevotella oralis, Prevotella nigrescens, Alloprevotella rava and Alloprevotella tannerae were associated with early RA independent of severity of periodontitis.

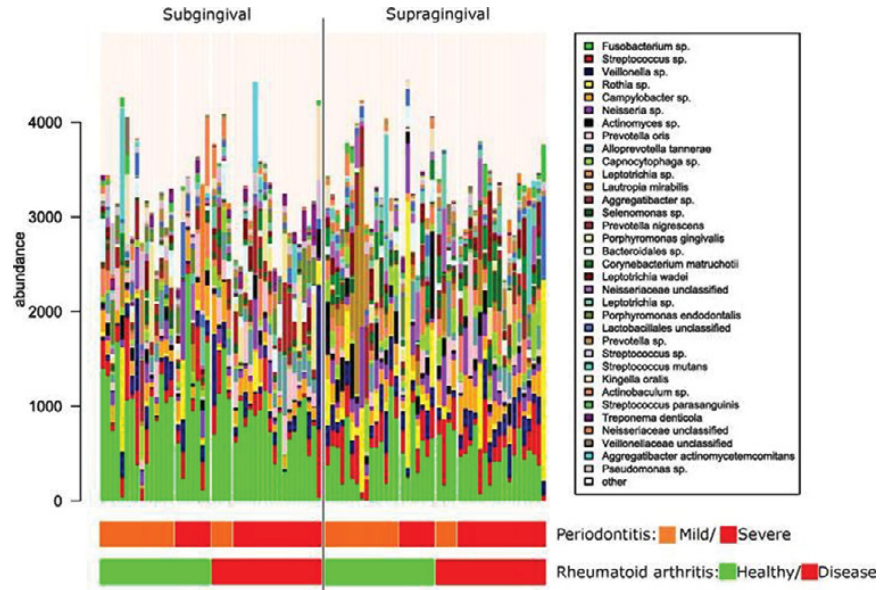

Conclusions: Prevotella and Alloprevotella species were enriched in patients with early RA independent of severity of periodontitis. Further studies are needed to test a causal relationship of these species with onset and/or disease progression of RA.

References:

[1] Oral status in patients with early rheumatoid arthritis: a prospective, casecontrol study. Wolff B, Berger T, Frese C, Max R, Blank N, Lorenz HM, Wolff D. Rheumatology (Oxford). 2014 Mar;53(3):526-31.

[2] The lung microbiota in early rheumatoid arthritis and autoimmunity. Scher JU, Joshua V, Artacho A, Abdollahi-Roodsaz S, Öckinger J, Kullberg S, Sköld M, Eklund A, Grunewald J, Clemente JC, Ubeda C, Segal LN, Catrina Al. Microbiome. 2016 Nov 17;4(1):60.

[3] Expansion of intestinal Prevotella copri correlates with enhanced susceptibility to arthritis. Scher JU, Sczesnak A, Longman RS, Segata N, Ubeda C, Bielski C, Rostron T, Cerundolo V, Pamer EG, Abramson SB, Huttenhower C, Littman DR. Elife. 2013 Nov 5

Acknowledgements: Wolff and Boutin contributed equally.

Disclosure of Interest: None declared

DOI: 10.1136/annrheumdis-2017-eular.2874

\section{FRI0699 DETERMINANTS OF 12-MONTHS PERSISTENCE IN ANKYLOSING SPONDYLITIS PATIENTS INITIATING SUBCUTANEOUS TNF-ALPHA INHIBITORS}

B. Fautrel $^{1}$, M. Belhassen ${ }^{2}$, C. Hudry ${ }^{3}$, M. $^{-C}$. Woronoff ${ }^{4}$, N. Gouyette ${ }^{5}$,

A. Clément ${ }^{5}$, E. Van Ganse ${ }^{6}$, F. Tubach ${ }^{7}$. ${ }^{1}$ Sorbonne Universités, UPMC Univ Paris 06; AP-HP, Rheumatology Department, Pitié Salpétrière University Hospital, Paris, France., Paris; ${ }^{2}$ Hesper 7425 , Health Services and Performance Research, University Claude Bernard Lyon 1; PELyon, Pharmacoepidemiologie Lyon, Lyon: ${ }^{3}$ AP-HP Hôpital Cochin, Paris; ${ }^{4} \mathrm{CHU}$ Besançon, Université Franche-Comté, Comue Ubfc, UMR INSERM 1098, Besançon; ${ }^{5}$ Merck Sharp \& Dohme, Paris; ${ }^{6}$ Hesper 7425, Health Services and Performance Research, University Claude Bernard Lyon 1, PELyon, Pharmacoepidemiologie Lyon, Lyon; ${ }^{7}$ APHP, Hôpital Pitié-Salpêtrière, Département de Biostatistiques, Santé publique et Information médicale, APHP, Centre de Pharmacoépidémiologie (Cephepi), INSERM, UMR 1123 ECEVE; Université Pierre et Marie Curie Paris 6, Sorbonne Universités, Paris, France

Background: Biotherapies such as subcutaneous tumour necrosis factor-alpha inhibitors (SC-TNFis) have transformed the management of inflammatory joint diseases such as ankylosing spondylitis (AS). The assessment of SC- TNFis persistence and its determinants is needed.

Objectives: The objective of this study was to describe treatment persistence in real-world settings, and identify the determinants of persistence among AS patients initiating treatment with an SC-TNFi.

Methods: The French national health insurance scheme database lists all outpatient and inpatient healthcare consumption for individuals covered by the general health insurance scheme. Using French claims data, AS was diagnosed using Long Term Disease status and hospital admission, based on ICD-10 codes. Patients were then identified through prescription filled for adalimumab (ADA), etanercept (ETA), certolizumab pegol (CZP) and golimumab (GLM) between 2012/07/01 and 2013/12/31. A patient was considered as non-persistent in the event of a prolonged interruption of the therapy lasting 91 days or more. Persistence was estimated with Kaplan Meier analysis. Determinants of persistence in the 12 months before initiation were identified using Cox models. Results: A total of 9,098 patients with AS were identified. In the descriptive analyse of the 12 months persistence, differences were observed for AS patients, with raw/non-adjusted persistence rates of $33.2 \%$ for CZP, $49.3 \%$ for ETA, $52.4 \%$ for ADA and $54.5 \%$ for GLM. Results of the Cox model are presented, including hazard ratio for biotherapy, adjusted on sex, age, socio-economic status, and criteria on disease severity. The variables biotherapy, socio-economic status and hospital admission for IRMD did not meet the proportionality hypothesis of risks, 